\title{
Correction and Addition to Szegö Kernels Associated with Discrete Series
}

\author{
Invent. math. 34, 163-200 (1976)
}

\author{
A.W. Knapp ${ }^{\star} \star$ and N.R. Wallach ${ }^{2} \star$ \\ ${ }^{1}$ Department of Mathematics, Cornell University, Ithaca, NY 14853, USA \\ ${ }^{2}$ Department of Mathematics, Rutgers University, New Brunswick, NJ 08903, USA
}

David Vogan has pointed out that Lemma 5.3 is incorrect, even for matrix groups, and therefore some changes are needed in the statements of the main theorems. The changes in question are not decisive, but we feel that the accurately stated versions of the theorems should be in the literature. Actually, when changes are needed, the new results yield more Szegö mappings than were originally predicted and in that sense represent an improvement of the original results. Vogan also suggested the statement below of Theorem $A$ as an approach to making the necessary changes.

To correct matters, delete Lemma 5.3 and introduce $M_{1}=M_{0}(F \cap T)$, where $F$ is the finite group defined in the proof of Lemma 5.3. Redefine $\sigma_{\lambda}$ on p. 176 to be the restriction of $\tau_{\lambda}\left(M_{1}\right)$ to the $M_{1}$-cyclic subspace $H_{\lambda}$ generated by $\phi_{\lambda}$. As in Proposition 5.5, we can conclude that $\sigma_{\lambda}$ is irreducible and has the stated highest weight and highest weight vector. The character $\xi_{\lambda}$ gives the values of $\sigma_{\lambda}$ on elements of $F \cap T$, instead of $F$. For the most part, we can then replace subsequent occurrences of $M$ by $M_{1}$ and of induction from $M A N$ by induction from $M_{1} A N$, and the results through the end of $\S 10$ go through, with their new interpretations. (At the beginning of $\S 8$, delete the fourth paragraph and then define $\Lambda(\sigma, v)$ directly in the obvious fashion.) No changes are needed in $\S \S 11-12$.

Qualitatively the result is that the Szegö mapping $f \rightarrow S f$ now operates on a different domain of functions but otherwise has the same properties as in Theorem 1.1. The new domain is smooth functions from $K$ into the redefined $H_{\lambda}$ that transform under the smaller group $M_{1}$ according to the redefined $\sigma_{\lambda}$. In representation-theoretic terms, the Szegö map $S$ gives an intertwining operator between a representation $W\left(\sigma_{\lambda}, 2 \rho^{+}-v\right)$ induced from $M_{1} A N$ to $G$ (rather than $M A N$ to $G$ ) and the discrete series $\pi_{A}$.

We can use this result to get an explicit quotient map to $\pi_{A}$ from a representation induced from $M A N$ to $G$. To this end, let

* Supported by the National Science Foundation 
$\left(\tilde{\sigma}_{\lambda}, \tilde{H}_{\lambda}\right)=$ representation of $M$ on the $M$-cyclic subspace of $\phi_{\lambda}$ in $V_{\lambda}$,

$$
\begin{aligned}
\tilde{\sigma}_{\dot{\lambda}} & =\sum_{j=1}^{r} \sigma_{j} \text { be a decomposition into irreducibles under } M, \\
\tilde{C}^{\infty}\left(K, \sigma_{j}\right) & =\left\{\begin{array}{ll}
f \in C^{\infty}\left(K, \text { space for } \sigma_{j}\right) & \begin{array}{l}
f(m k)=\sigma_{j}(m) f(k) \\
\text { for } m \in M, k \in K
\end{array}
\end{array}\right\},
\end{aligned}
$$

$U\left(\sigma_{j}, v\right)=$ induced representation of $G$ (in nonunitary principal series) nonunitarily induced from $\sigma_{j} \otimes v \otimes 1$ on $M A N$ (cf. formulas (6.6) and (6.7)), $M_{2}=\left\{m \in M \mid \tau_{\lambda}(m) H_{\lambda} \subseteq H_{\lambda}\right\}$.

Theorem A. With $\lambda=\Lambda+\delta_{n}-\delta_{k}$ integral and with $A$ nonsingular and G-dominant, the operator

$$
S_{j}(f)(x)=\int_{K} \tau_{\lambda}(k)^{-1} f(k x) d k=\int_{K} e^{\nu H\left(l x^{-1}\right)} \tau_{\lambda}\left(\kappa\left(l x^{-1}\right)\right)^{-1} f(l) d l
$$

carries $\tilde{C}^{\infty}\left(K, \sigma_{j}\right)$ into the kernel of the operator $\mathscr{D}$ on $C^{\infty}\left(G, \tau_{\lambda}\right)$, and under the identification of $\tilde{C}^{\infty}\left(K, \sigma_{j}\right)$ with the space of the nonunitary principal series $U\left(\sigma_{j}, 2 \rho^{+}-v\right)$, it carries the $K$-finite vectors of $U\left(\sigma_{j}, 2 \rho^{+}-v\right)$ in a g-equivariant fashion onto the $K$-finite vectors of the discrete series $\pi_{A}$.

Proof. Clearly $S_{j}$ is g-equivariant. Define a function $f_{j}$ in $\tilde{C}^{\infty}\left(K, \sigma_{j}\right)$ by $f_{j}(k)$ $=P_{\sigma_{j}} \tau_{\lambda}(k) \phi_{\lambda}$, where $P_{\sigma_{j}}$ is the orthogonal projection on the space for $\sigma_{j}$. Then

$$
S_{j} f_{j}(1)=\int_{K} \tau_{\lambda}(k)^{-1} P_{\sigma_{j}} \tau_{\lambda}(k) \phi_{\lambda} d k=\frac{\text { Trace } P_{\sigma_{j}}}{\text { degree } \tau_{\lambda}} \phi_{\lambda}=\frac{\text { degree } \sigma_{j}}{\text { degree } \tau_{\lambda}} \phi_{\lambda} .
$$

Thus $S_{j}$ is not the 0 map. In view of Proposition 10.7, Theorem 10.8, and the remark after Theorem 10.8, Theorem A will follow if we show that image $S_{j} \subseteq$ image $S$.

Let $\left\{q_{i}, 1 \leqq i \leqq n\right\}$ be representatives of $M / M_{2}$ chosen from $F$. Formula (3) below, valid in the linear case, implies here that $F$ normalizes $T$. Thus each $q_{i}$ gives rise to a member $s_{i}$ of the Weyl group $W_{K}$, and we have $\tau_{\lambda}\left(q_{i}\right) \phi_{\lambda}=c_{i} \phi_{s_{i} \lambda}$. These $s_{i} \lambda$ are distinct, $1 \leqq i \leqq n$; in fact, $s_{i} \lambda=s_{j} \lambda$ leads to $\tau_{\lambda}\left(q_{i}^{-1} q_{j}\right) \phi_{\lambda}=c \phi_{\lambda}$, hence $q_{i}^{-1} q_{j} \in M_{2}$, and hence $q_{i}=q_{j}$. Consequently the vectors $\tau_{\lambda}\left(q_{i}\right) \phi_{\lambda}, 1 \leqq i \leqq n$, are linearly independent. Each such is a highest weight vector for $M_{0}$, since $\operatorname{Ad}(F)$ acts on $m$ as the identity, and thus

$$
\operatorname{dim}\left(\sum_{i=1}^{n} \tau_{\lambda}\left(q_{i}\right) H_{\lambda}\right) \geqq n \operatorname{dim} H_{\lambda}=\sum_{i=1}^{n} \operatorname{dim} \tau_{\lambda}\left(q_{i}\right) H_{\lambda} .
$$

Consequently the spaces $\tau_{\lambda}\left(q_{i}\right) H_{\lambda}$ are independent and

$$
h_{i} \in H_{\lambda} \quad \text { for } 1 \leqq i \leqq n \text { and } \quad \sum_{i=1}^{n} \tau_{\lambda}\left(q_{i}\right) h_{i}=0
$$

imply $h_{i}=0$ for all $i$.

From (1) it follows that the operator $T$ on $\tilde{H}_{\lambda}$ given by

$$
T=\sum_{i=1}^{n} \tau_{\lambda}\left(q_{i}\right) P \tau_{\lambda}\left(q_{i}\right)^{-1},
$$


where $P$ is the orthogonal projection of $\tilde{H}_{\lambda}$ on $H_{\lambda}$, is invertible. In fact, if $T v=0$, take $h_{i}=P \tau_{\lambda}\left(q_{i}\right)^{-1} v$ in (1) to see that $P \tau_{\lambda}\left(q_{i}\right)^{-1} v=0$ for all $i$. In terms of the inner product in $\hat{H}_{\lambda}$, we then have

$$
\begin{array}{rlrl}
\left\langle\tau_{\lambda}\left(q_{i}\right)^{-1} v, u\right\rangle=0 & \text { for all } u & \text { in } H_{\lambda} \text { and all } i, \\
\left\langle\tau_{\lambda}\left(q_{i}\right)^{-1} v, \tau_{\lambda}\left(m_{2}\right) \phi_{\lambda}\right\rangle=0 & \text { for all } m_{2} \text { in } M_{2} \text { and all } i, \\
\left\langle v, \tau_{\lambda}\left(q_{i} m_{2}\right) \phi_{\lambda}\right\rangle=0 & \text { for all } m_{2} \text { in } M_{2} \text { and all } i, \\
\left\langle v, \tau_{\lambda}(M) \phi_{\lambda}\right\rangle=0, & &
\end{array}
$$

and so $v=0$ since $\phi_{i}$ is $M$-cyclic in $\tilde{H}_{\lambda}$.

Thus $T$ is invertible on $\tilde{H}_{\lambda}$. But also $T$ commutes with all $\tilde{\sigma}_{\lambda}(m)$ for $m$ in $M$ since

$$
\int_{M} \tau_{\lambda}(m) P \tau_{\lambda}(m)^{-1} d m=\sum_{i=1}^{n} \tau_{\lambda}\left(q_{i}\right) P \tau_{\lambda}\left(q_{i}\right)^{-1}=T .
$$

Let $f$ in $\tilde{C}^{\infty}\left(K, \tilde{\sigma}_{\lambda}\right)$ be given, and define $F=T^{-1} \circ f$. Then it follows that $F$ is in $\tilde{C}^{\infty}\left(K, \tilde{\sigma}_{\lambda}\right)$ and

$$
\begin{aligned}
\int_{M} \tau_{\lambda}(m)^{-1} P F(m k) d m & =\left(\int_{M} \tau_{\lambda}(m)^{-1} P \tau_{\lambda}(m) d m\right) F(k) \\
& =T(F(k))=f(k) .
\end{aligned}
$$

Now $P \circ F$ is in $C^{\infty}\left(K, \sigma_{\lambda}\right)$ since $P \tilde{\sigma}_{\lambda}\left(m_{2}\right)=\sigma_{\lambda}\left(m_{2}\right) P$ for $m_{2}$ on $M_{2}$. Consequently

$$
\begin{aligned}
S_{j}(f)(k) & =\int_{K \times M} S(x, k) \tau_{\lambda}(m)^{-1} P \circ F(m k) d m d k \\
& =\int_{K \times M} S(x, m k) P \circ F(m k) d m d k \\
& =\int_{K} S(x, k) P \circ F(k) d k \quad \text { after } m k \rightarrow k \\
& =S(P \circ F)(k)
\end{aligned}
$$

and image $S_{j} \subseteq$ image $S$. This proves Theorem A.

In short, each irreducible constituent of $\tilde{\sigma}_{\lambda}$ leads to a Szegö mapping whose image is the same discrete series. In the linear case we can say more. The group $F$ is central in $M$ and is spanned by the commuting elements $\gamma_{\beta}$ of order at most 2 given by

$$
\gamma_{\beta}=\exp 2 \pi i|\beta|^{-2} h_{\beta},
$$

where $\beta$ runs through the restricted roots and $h_{\beta}$ is the member of a dual to $\beta$. (See [26], p. 93.) Thus $F$ is a sum of copies of $\mathbb{Z}_{2}$, and $M$ is the direct sum of $M_{2}$ and a group $\sum \mathbb{Z}_{2}$. It follows that $\tilde{\sigma}_{\lambda}$ is multiplicity-free and that the number of distinct constituents $\sigma_{j}$ is $\left|M / M_{2}\right|$. The various $\sigma_{j}$ 's are related as follows: They have a common formula on $M_{2}$, and all of them are obtained from one of them on $\sum \mathbb{Z}_{2}$ by multiplying by an arbitrary character of $\sum \mathbb{Z}_{2}$. Theorem $\mathrm{B}$ below identifies the formula on $M_{2}$. Let $u=\Pi u_{\alpha_{j}}$ be the Cayley transform in Eq. (5.7), and let $\bar{\lambda}=\lambda \circ \operatorname{Ad}(u)$ and $\bar{\alpha}_{j}=\alpha_{j} \circ \operatorname{Ad}(u)$. 
Theorem B. Suppose $G$ is a matrix group and $\lambda$ is integral and $K$-dominant. If the product $\gamma=\gamma_{\beta_{1}} \ldots \gamma_{\beta_{q}}$ is in $F \cap M_{2}$, then $\tilde{\sigma}_{\lambda}(\gamma)$ acts on $\tilde{H}_{\lambda}$ as the scalar -1 raised to the power

$$
\sum_{k} 2\left\langle\bar{\lambda}, \beta_{k}\right\rangle /\left|\beta_{k}\right|^{2}
$$

Proof. Since $\gamma$ is in $M_{2}, \tilde{\sigma}_{\lambda}(\gamma)$ leaves $H_{\lambda}$ stable. Since $\gamma$ is central in $M,\left.\tilde{\sigma}_{\sigma}(\gamma)\right|_{H_{\lambda}}$ commutes with $\sigma_{\lambda}\left(m_{1}\right)$ for $m_{1}$ in $M_{1}$. The irreducibility of $\sigma_{\lambda}$ implies that $\sigma_{\lambda}(\gamma)$ is scalar on $H_{\lambda}$, hence on $\phi_{\lambda}$. Since $\phi_{\lambda}$ is $M$-cyclic for $\tilde{\sigma}_{\lambda}$ on $\tilde{H}_{\lambda}$ and since $\gamma$ is central in $M, \tilde{\sigma}_{\lambda}(\gamma)$ is scalar on $\tilde{H}_{\lambda}$. Thus it is enough to identify the scalar $c$ in the equation

$$
\tau_{\lambda}(\gamma) \phi_{\lambda}=c \phi_{\lambda}
$$

We need a different formula for $\gamma$. If $p_{\alpha}$, denotes a particular one of the two standard representatives of the reflection in $\alpha_{j}$ in the Weyl group $W_{K}$, then we shall show that

$$
\gamma_{\beta}=\prod_{j} p_{\alpha_{j}}^{2\left\langle\beta, \vec{\alpha}_{j}\right\rangle /\left|\tilde{x}_{j}\right|^{2}}
$$

In fact, our definitions make $h_{\alpha_{j}}=\operatorname{Ad}\left(u_{\alpha_{j}}\right)^{-1} H_{\alpha_{j}}$. We can expand

$$
h_{\beta}=\sum \frac{\left\langle\beta, \bar{\alpha}_{j}\right\rangle}{\left|\bar{\alpha}_{j}\right|^{2}} h_{\bar{\alpha}_{j}}
$$

and then

$$
\begin{aligned}
\gamma_{\beta} & =\exp \sum_{j} \frac{2\left\langle\beta, \bar{\alpha}_{j}\right\rangle}{|\beta|^{2}} \frac{\pi i h_{\alpha_{j}}}{\left|\bar{\alpha}_{j}\right|^{2}}=\prod_{j}\left(\exp \frac{\pi i h_{\bar{\alpha}_{j}}}{\left|\bar{\alpha}_{j}\right|^{2}}\right)^{\left.2\left\langle\beta, \bar{\alpha}_{j}\right\rangle\right\rangle|\beta|^{2}} \\
& =\prod_{j} \exp \left(\frac{1}{2} \pi i\left(E_{\alpha_{j}}+E_{-\alpha_{j}}\right)\right)^{\left.2\left\langle\beta, \bar{\alpha}_{j}\right\rangle\right\rangle|\beta|^{2}}=\prod_{j} p_{\alpha_{j}}^{2\left\langle\beta, \bar{\alpha}_{j}\right\rangle|\beta|^{2}}
\end{aligned}
$$

as required. This proves (3).

Since $\lambda$ is integral and $G$ is a matrix group, we can introduce a $G$-ordering (for current purposes) so that $\lambda$ is $G$-dominant. Let $\tilde{\tau}_{\lambda}$ be an irreducible representation of $G$ with highest weight $\lambda$. Then it is easy to see that the restriction of $\tilde{\tau}_{\lambda}(K)$ to the span of a highest weight vector is equivalent with $\tau_{\lambda}$. That is, we may regard $\tau_{\lambda}$ as extended from $K$ to $G$, with the space suitably enlarged.

In view of (3), $\tau_{\lambda}(\gamma) \phi_{\lambda}$ is a weight vector for the weight

$$
\left(\prod_{j, k} p_{\alpha_{j}}^{\left.\left.2\left\langle\beta_{k}, \bar{\alpha}_{j}\right\rangle|| \bar{\alpha}_{j}\right|^{2}\right) \lambda .}\right.
$$

Hence (2) implies that

$$
\left\langle\lambda, \alpha_{j}\right\rangle=0 \quad \text { whenever } \sum_{k} \frac{2\left\langle\beta_{k}, \bar{\alpha}_{j}\right\rangle}{\left|\bar{\alpha}_{j}\right|^{2}} \text { is odd }
$$

and hence that $\tau_{\lambda}\left(p_{\alpha}\right)$ fixes $\phi_{\lambda}$ for these $j$. For any $j$,

$$
\begin{aligned}
\tau_{\lambda}\left(p_{\alpha_{j}}\right)^{2} \phi_{\lambda} & =\tau_{\lambda}\left(\gamma_{\alpha_{j}}\right) \phi_{\lambda} \\
& =(-1)^{\left.2\left\langle\lambda, \alpha_{j}\right\rangle|| \alpha_{j}\right|^{2}} \phi_{\lambda}=(-1)^{\left.2\left\langle\bar{\lambda}_{,}, \bar{\alpha}_{j}\right\rangle|| \alpha_{j}\right|^{2}} \phi_{\lambda}
\end{aligned}
$$


by a computation in $S L(2, \mathbb{R})$. Then (4) and (5) show that $c$ in (2) is given by -1 raised to the power

$$
\sum \frac{2\left\langle\bar{\lambda}, \bar{\alpha}_{j}\right\rangle}{\left|\bar{\alpha}_{j}\right|^{2}} \frac{\left\langle\beta_{k}, \bar{\alpha}_{j}\right\rangle}{\left|\beta_{k}\right|^{2}}
$$

with the sum extended over those $j$ for which $\sum_{k} 2\left\langle\beta_{k}, \bar{\alpha}_{j}\right\rangle /\left|\bar{\alpha}_{j}\right|^{2}$ is even. The sum in (6) may be further extended to be over all $j$ because of (4), and then (6) reduces to the sum in the statement of the theorem.

Theorem C. If $G$ is a matrix group and $\lambda$ is integral and $K$-dominant, then $2\langle\bar{\lambda}, \beta\rangle /|\beta|^{2}$ is an integer for every restricted root $\beta$, and the assignment

$$
\sigma\left(\gamma_{\beta}\right)=(-1)^{2\langle\bar{\lambda}, \beta\rangle /|\beta|^{2}}
$$

extends to a well-defined character of $F$. Moreover, some constituent $\sigma_{1}$ of $\tilde{\sigma}_{\lambda}$ has $\sigma_{1}(z)=\sigma(z) I$ for all $z$ in $F$.

Proof. Since $\lambda$ is integral, $\lambda$ is the differential of a well-defined character $\xi_{\lambda}$ on $T$. Define a character $\sigma$ on $\exp i a \subseteq G^{\mathbb{C}}$ by $\sigma(z)=\xi_{\lambda}\left(u z u^{-1}\right)$. Applying (3), we have

Therefore

$$
\begin{aligned}
u \gamma_{\beta} u^{-1} & =\prod_{j} \exp \left(\frac{1}{2} \pi i \operatorname{Ad}(u)\left(E_{\alpha_{j}}+E_{-\alpha_{j}}\right)\right)^{\left.2\left\langle\beta, \bar{\alpha}_{j}\right\rangle|| \beta\right|^{2}} \\
& =\prod_{j} \exp \left(\pi i\left|\alpha_{j}\right|^{-2} H_{\alpha_{j}}\right)^{2\left\langle\beta, \bar{\alpha}_{j}\right\rangle|\beta|^{2}} .
\end{aligned}
$$

$$
\begin{aligned}
\sigma\left(\gamma_{\beta}\right) & =\xi_{\lambda}\left(u \gamma_{\beta} u^{-1}\right) \\
& =\exp \left(\pi i \sum_{j} \frac{\left\langle\lambda, \alpha_{j}\right\rangle}{\left|\alpha_{j}\right|^{2}} \frac{2\left\langle\beta, \bar{\alpha}_{j}\right\rangle}{|\beta|^{2}}\right) \\
& =\exp \left(\pi i \sum_{j} \frac{\left\langle\bar{\lambda}, \bar{\alpha}_{j}\right\rangle}{\left|\bar{\alpha}_{j}\right|^{2}} \frac{2\left\langle\beta, \bar{\alpha}_{j}\right\rangle}{|\beta|^{2}}\right) \\
& =\exp \left(\pi i \frac{2\langle\bar{\lambda}, \beta\rangle}{|\beta|^{2}}\right) .
\end{aligned}
$$

Since $\gamma_{\beta}^{2}=1$, it follows that $2\langle\bar{\lambda}, \beta\rangle /|\beta|^{2}$ is an integer.

Now write $M=M_{2} \oplus \sum \mathbb{Z}_{2}$ with $\sum \mathbb{Z}_{2} \subseteq F$. Let $\sigma_{0}$ be a constituent of $\tilde{\sigma}_{\lambda}$ and define

$$
\sigma_{1}(m)= \begin{cases}\sigma_{0}(m) & \text { for } m \in M_{2} \\ \sigma(m) I & \text { for } m \in \sum \mathbb{Z}_{2} .\end{cases}
$$

The remarks before Theorem B show that $\sigma_{1}$ is a constituent of $\tilde{\sigma}_{\lambda}$, and Theorem B shows that $\sigma_{1}(z)=\sigma(z) I$ for all $z$ in $F$.

Concluding Remarks. 1) If $G$ is a matrix group and $\lambda$ is also $K$-regular, Theorem A gives $\left|M / M_{1}\right|$ distinct explicit quotient mappings. For $K$-singular $\lambda$, Theorem A gives only the smaller number $\left|M / M_{2}\right|$ of distinct explicit quotient mappings. However, an argument with tensor products on the nonunitary principal series and on $\mathscr{P}^{\lambda}$ shows the existence of $\left|M / M_{1}\right|$ maps from nonunitary 
principal series to $\mathscr{P}^{\lambda}$ even if $\lambda$ is $K$-singular; it is just that not all of these maps are given by Theorem $\mathrm{A}$.

2) Vogan offered $S O(4,4)$ as a counterexample to Lemma 5.3. For other groups it is often the case that $M_{1}=M$, hence that Lemma 5.3 remains correct. This happens for $G$ if it happens for the adjoint group of $G$. It happens if $G$ has real-rank one, or if $M$ is connected (e.g., when the restricted roots form a $B C$ diagram), or if $G / K$ is Hermitian symmetric. Among the classical simple groups, it can fail only for groups locally isomorphic to $S O(m, n)$.

\section{Reference}

26. Satake, I.: On representations and compactifications of symmetric Riemannian spaces. Ann. of Math. 71, 77-110(1960)

Received July 16, 1980 